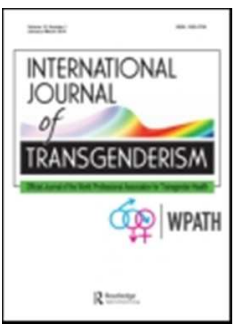

\title{
A comparison of mental health symptomatology and levels of social support in young treatment seeking transgender individuals who identify as binary and non-binary.
}

\begin{tabular}{|r|l|}
\hline Journal: & International Journal of Transgenderism \\
\hline Manuscript ID & WIJT-2017-0096.R1 \\
\hline Manuscript Type: & Original Research \\
\hline Keywords: & $\begin{array}{l}\text { Gender Dysphoria, Transgender, Non-binary, Youth, Anxiety, Depression, } \\
\text { Non-suicidal self-injury, Self-esteem }\end{array}$ \\
\hline
\end{tabular}

SCHOLARONE ${ }^{\text {M }}$

Manuscripts 


\begin{abstract}
ABSTACT
Background: Previous research has consistently reported high rates of mental health symptomatology and lower social support in young treatment seeking transgender individuals. However, these studies have failed to distinguish between transgender people who identify within the gender binary and those who identify as non-binary.

Aims: This study aimed to compare levels of mental health symptomatology (anxiety, depression, and non-suicidal self-injury behaviour) and social support of treatment seeking non-binary transgender young individuals with those self-identified as binary transgender young individuals. All participants were attended a national transgender health service in the UK during a 2 years period.
\end{abstract}

Measures: Age and gender identity descriptors were collected, as well as clinical measures of anxiety and depression (Hospital Anxiety and Depression Scale), self-esteem (The Rosenberg Self-Esteem Scale), non-suicidal self-injury (Non-Suicidal Self-Injury: Treatment Related), and social support (Multidimensional Scale of Perceived Social Support).

Results: A total of 388 young people, aged 16-25 years, agreed participation; $331(85.3 \%)$ identified as binary and $57(14.7 \%)$ as non-binary. Analysis of the data showed the nonbinary group experienced significantly more anxiety and depression and had significantly lower self-esteem that the binary group. There were no significant differences between groups in the likelihood of engaging in non-suicidal self-injury behaviour or levels of social support.

Conclusions: Non-binary identifying treatment seeking transgender youth are at increased risk of developing anxiety, depression and low self-esteem compared to binary transgender youth. This may reflect the even greater barriers and feelings of discrimination that may be faced by those whose identity does not fit the notion of binary gender that is pervasive in how society views both cis- and transgender populations.

Keywords: Gender Dysphoria, Trans, Transgender, Non-binary, Youth, Anxiety, Depression, Non-suicidal self-injury, Self-esteem, Social Support. 


\section{Introduction}

Over recent years, the number of people identifying as transgender and being referred to transgender health services has increased rapidly (Arcelus et al, 2015; Collin, Reisner, Tangpricha \& Goodman, 2016; Zucker, 2017). Particularly visible within this population are individuals who express the feeling of laying outside of the established gender roles of 'male' and 'female'. Within this group, gender identity is experienced as neither exclusively female or male but as a combination or total absence of binary gender identification which may vary or stay stable over time. One of the accepted terms for this group, and the one which will be used throughout this paper, is 'non-binary'. In Richards, Bowman and Barker (2017) this is described as 'people who identify as a single fixed gender position other than male or female'. This includes those who are 'genderqueer', those who have no gender and those who totally reject the very idea of gender altogether. A study in the UK found that $5 \%$ of lesbian, gay, bisexual, trans and questioning (LGBTQ) youth did not identify as either male or female (METRO Youth Chances, 2014). However, not every non-binary individual will access transgender health services, as many will not require transition-related medical interventions. This also applies to those who do identify with a binary gender. It is also likely that those accessing services may present with higher dysphoria about their bodies than those not accessing them, although studies have not investigated this (Witcomb et al,. 2918).

The vulnerability of treatment seeking transgender individuals to mental health problems, particularly anxiety, depression and non-suicidal self-injury behavior has been well established in a number of studies (Bouman et al., 2017; Claes et al., 2015; Dhejne et al., 2015; Kessler, Berglund, Demler, Jin, \& Walters, 2005; Marshall et al., 2016; Millet Longworth, \& Arcelus, 2017; Nuttbrock, Rosenblum, \& Blumenstein, 2002; Heylens et al, 2014). In particular, the rates of anxiety and depression symptomatology in the transgender community as a whole has consistently been found to be high (Dhejne et al., 2015). For example, Budge, Andelson and Howard (2013) reported that in their non-clinical sample of 226 transgender women and 125 transgender men, clinical levels of anxiety were found in $40.4 \%$ of the transgender women and $47.5 \%$ of the transgender men, and clinical levels of depression were found in $51.4 \%$ of the transgender women and $48,3 \%$ of the transgender men. This is a higher rate than would be expected in a general population sample (Office for 
National Statistics, 2017). However, it should be recognised that large-scale studies of the general population will also inevitably also include transgender individuals even if they are not acknowledged specifically in the data analysis. Bouman et al. (2017) compared anxiety symptomatology levels in a sample of treatment seeking transgender individuals and a control sample from the general population, matched on age and gender, and found that transgender individuals had a near threefold increased risk of clinical anxiety (assessed using Hospital Anxiety and Depression Scale, also known as HADS) compared to the general population sample. Non-suicidal self-injury behavior has also been found to be significantly more prevalent in the transgender community compared to the general population (Davey, Arcelus, Meyer \& Bouman, 2016). Indeed, a recent systematic literature review found a strong association between gender dysphoria and non-suicidal self-injury (Marshall, Claes, Bouman, Witcomb \& Arcelus, 2016), a finding supported by a study of 268 transgender youth that reported that $46.3 \%$ had previously or were currently engaging in non-suicidal self-injury behaviors (Arcelus, Claes, Witcomb, Marshall \& Bouman, 2016), slightly more than the rate of $36.8 \%$ (of a sample of 155) reported in older transgender adults (Claes et al., 2015).

Parallel to the increasing number of people identifying as transgender, research in the field of transgender health is growing rapidly. Studies investigating the predictors of mental health symptomatology offer insights into the factors that are underpinning distress and difficulties and are therefore important factors to target for interventions. For example, interpersonal difficulties (Arcelus et al., 2016) and lack of social support (Davey et al., 2015, Bouman et al., 2015) have been repeatedly shown to predict poor mental health. Social support for some transgender people may well be lacking due to prejudice and rejection of the new identity by family members, which could also contribute to higher rates of poor mental health overall. Age has also been found to be important, with younger transgender individuals often reporting more difficulties than older participants (Arcelus et al, 2016, Rimes et al, 2017), highlighting the need to ensure that studies adequately consider age as a variable in the methodological design or statistical analysis. As a result, more recent studies have started to focus specifically on transgender youth. While recent research is benefitting from more rigorous methodological approaches, a factor that has been largely unexplored is the extent to which a transgender person identifies within a binary or non-binary system. Thus, a limitation of most studies is that they do not specifically distinguish between individuals identifying as non-binary and those identifying as binary. Especially, research drawing upon clinical 
samples is limited in terms of the visibility of non-binary transgender individuals (Eyssel et al, 2017)

In line with recently revised diagnoses (DSM-5: Gender Dysphoria, ICD-11 draft: Gender Incongruence), covering a spectrum of diverse gender-related issues (e.g., non-binary genders), clinical approaches of transgender health services broadened their range of genders accepted, both binary and non-binary. However, since non-binary identities have not received sufficient attention so far, neither empirically nor clinically, the increasing number of treatment seeking non-binary transgender individuals is still under diagnostic pressure to present within the gender binary due to a lack of scientific and clinical knowledge. However, it is very likely that non-binary individuals have always been there without disclosing their unique gender experience (Nieder \& Richter-Appelt, 2011) and have been treated "without being distinguished as such" (Hage \& Karim, 2000). This is problematic, both empirically and clinically, for two reasons. Firstly, it assumes that the issues faced by binary and nonbinary transgender individuals are largely the same. However, in much the same way that studies on LGBT populations that do not address the abundant differences between LBG and T populations can be criticised (Dargie, Blair, Pukall, \& Coyle, 2014), the assumption that binary and non-binary individuals are a homogenous group is equally unacceptable. While there is no doubt that many areas of society struggle to accept transgender individuals (Stocks, 2015), the majority of the population can at least identify with the gender binary of which many transgender people identify (Wiseman \& Davidson, 2012). For non-binary transgender individuals, there is an additional stigma of falling outside of the societal norm altogether, leading to complications with no clear resolution, such as pronouns, titles on official documentation and issues of which gendered toilet to use (Richards, 2017).

Secondly, it is likely that many studies of transgender populations have indeed included nonbinary transgender individuals, but that these participants have been subsumed within the wider bracket of 'trans'. Thus, any conclusions drawn about the mental health and wellbeing of transgender individuals may be inflated by the (unreflected and/or unknown) presence of non-binary transgender individuals who may differ in their levels of symptomatology.

Therefore, the aim of this study was to address these limitations and to explore differences in mental health symptomology (anxiety, depression), non-suicidal self-injury, self-esteem and 
social support between young treatment seeking transgender individuals identifying as binary and those who identify as non-binary.

\section{$\underline{\text { Method }}$}

\section{$\underline{\text { Participants }}$}

All individuals, aged 16-25 years, who were referred for an assessment at a national transgender health service in the United Kingdom during a 2-year period, between June 2015 and June 2017, were invited to participate in the study. The age range starts at 16 as this was the minimum age required for an appointment at the time the data was collected. The clinic is one of the largest of its kind in Europe and receives referrals of people with a transgender identity aged 17 and over. At the time of data collection, this age was 16 but has since been raised to 17 .

\section{Procedure}

Prior to their first assessment appointment at the service, participants were invited to complete a questionnaire pack containing a number of forms to record socio-demographic information and a number of questionnaires assessing health and wellbeing. This information is part of a longitudinal study investigating the outcome of gender affirming treatment. Participants' informed consent was obtained in order for their data to be used for research purposes. The study received ethical approval from the NHS Ethics committee and from the Research and Development Department from the Nottinghamshire Healthcare NHS Foundation Trust in line with Health Research Authority guidance (HRA, 2013). At this stage, those who reported that they were already receiving any form of hormone treatment were excluded from the study.

\section{Main outcome variables}

Demographic information. Participants were invited to complete a set of questions recording information on age, ethnicity, employment status, civil status, and living situation. In addition, they were asked to indicate how they describe their gender identity (e. g., I identify as a man; I identify as a woman; I identify partly as a man, and partly as a woman; I 
identify neither as a man; nor as a woman; I don't know what my gender identity is (yet) or I am questioning my gender identity, and other) and how they identify themselves (e. g., Trans*, Gender neutral / neutrois, Trans, Non-binary gender, Transvestite, Pangender, Transgender, Bigender, Transsexual, Genderqueer, Androgynous, I don't know, Other). The range of responses available had been developed in consultation with transgender people and other stakeholders as part of another study funded by the World Health Organization (see Beek et al., 2016; 2017 for full descriptors).

Hospital Anxiety and Depression Scale (HADS; Zigmond \& Snaith, 1983). This is a 14item self-report screening scale that was originally developed to indicate the possible presence of anxiety and depression states in the setting of a medical non-psychiatric outpatient clinic. HADS consists of two subscales, HAD-Anxiety (HAD-A) and HADDepression (HAD-D), each with seven items. An example of an anxiety question is "I feel tense or "wound up" "and an example of a depression question is "I feel as if I am slowed down". Participants are asked to indicate, using a 4-point Likert scale indicating agreement or disagreement with the items (e.g. Most of the time; A lot of the time; Time to time, occasionally; Not at all) how often they have felt that way during the preceding week. A score of 0-7 on either scale is regarded as being in the normal range (no symptoms), a score of 8-10 is suggestive of the presence of an anxiety or depressive disorder (possible symptoms), and a score of 11 or higher indicates the probable presence of an anxiety or depressive disorder (symptoms). Maximum subscales scores are 21. The HADS was found to perform well in assessing the symptom severity and caseness of depressive disorders in both somatic, psychiatric and primary care patients and in the general population (Bjelland et al., 2002) and it has been previously used with transgender individuals (Bouman et al., 2016a; Gomez-Gil et al., 2012). In this study, the Cronbach's alpha was 0.83 for the anxiety scale and 0.71 for the depression scale

Non Suicidal Self-Injury (NSSI) Questionnaire -Treatment Related (SIQ-TR; Claes \& Vandereycken 2007). The SIQ-TR measures types, duration, frequency, and emotions related to non-suicidal self-injury (NSSI), which is the intentional injuring of body tissue, but with the absence of suicidal motivations. For this study, participants were only asked to indicate whether they had engaged in any form of NSSI (cut/bruise/bite/burn/scratch/other method of self-injury) and if so, how long ago (more than a year ago, several months ago, within the last month, within the last week). For this study, the results were dichotomized to simply describe 
Rosenberg Self Esteem Scale (RSE; (Rosenberg, 1965). This is a self-report measure of global self-esteem. Examples of items include “At times, I think I am no good at all” and "I feel that I'm a person of worth, at least on an equal plane with others". Responses are rated on a 4-point rating scale ranging from 0 to 3 . The total score is calculated by summing the item scores, with some items reversed scored to ensure higher scores indicate higher selfesteem. The RSE has received extensive empirical validation (Robins et al., 2001) and has been used previously with transgender individuals (e.g., Vocks et al., 2009; Skrapec \& MacKenzie, 1981). For this study the Cronbach alpha was 0.89 .

Multidimensional Scale of Perceived Social Support (MSPSS; Zimet et al. 1988). This 12item questionnaire assesses the respondent's perception of social support across three facets: family, friends, and significant others. Example questions include "My family really tries to help" and "I can talk about my problems with my friends". All items are rated from 1 (very strongly disagree) to 7 (very strongly agree). A total mean score is the calculated, with lower scores indicating lower support. The MSPSS has well documented psychometric properties (Cecil et al. 1995; Clara et al. 2003; Dahlem et al. 1991) and has been used in transgender populations (Davey et al., 2016; Meier et al., 2013;). The Cronbach's alpha for the subscales and the total MSPSS ranged between 0.84 and 0.94 .

\section{$\underline{\text { Data Analysis }}$}

All data was tabulated and analysed using SPSS 22 (IBM, 2013). The scores of the HADS, RSE, SQR-TR, and MSPSS were positively skewed in both groups and non-normally distributed, as demonstrated by significant $(p<.002)$ Kolmogorov-Smirnov tests and ShapiroWilk tests; therefore, for comparisons of the means of the scores on the aforementioned questionnaires between the two groups, as well as age differences, Mann-Whitney U tests were conducted. Chi-square tests were used to test for any differences in assigned sex between the binary and non-binary groups. No violation of the assumption of homogeneity of 
variance was detected using a Levene's test (p>.05). In view of this, in spite of having a larger number of participants in one group (binary) compared to the other (non-binary), it was not deemed necessary to match the groups by age and gender as in previous studies (Arcelus et al., 2016). The level of significance used was $p<0.05$.

\section{$\underline{\text { Results }}$}

\section{$\underline{\text { Participants }}$}

During the study period a total of 703 people were invited for assessment to the service. More than half $(59.1 \%, \mathrm{n}=416)$ were within the age range for the study (aged 25 years old or younger). Of these, $28(6.7 \%)$ declined to participate, leading to a sample of $N=388$ treatment seeking transgender individuals $\geq 25$ years.

Participants were categorised as having either a binary gender or a non-binary gender based on their responses to a demographic questionnaire. Most participants had clearly selected a non-binary or binary gender option on the form. In some cases, there was less clarity with a number of participants choosing binary gender options such as 'I am a male', but later in the form identifying themselves as more ambivalent in their gender, such as 'I am not sure of my gender identity yet'. In these cases, these people were categorised as non-binary due to not clearly identifying on the gender binary. Out of the 388 participants, 57 (14.7\%) were categorized as non-binary and the remaining 331 were classified as binary $(85.3 \%)$.

\section{Age and self-reported gender description of the two groups.}

The mean age for the sample was 20.16 years $(S D=2.69$, Median=20/00, IQR $=4)$, with an age range of 16 to 25 years. A Mann-Whitney $U$ test found that the binary group was significantly younger $($ mean $=20.02$ years, $[S D=2.71])$ than the non-binary group $($ mean $=$ $21.02[S D=0.354] ; U=7269, z=-2.79, p=0.005)$. The effect size of these two groups $(\mathrm{d}=$ 0.517) showed a medium effect according to Cohen's convention (1988). Analysis of the distribution of assigned sex and binary / non-binary identification found that in the binary group, $55.5 \%$ were birth assigned females $(n=184)$ and $44.4 \%$ were birth assigned males $(n=147)$. The percentages were similar in the non-binary group, with $50.1 \%$ of participants 
reporting being assigned female at birth $(n=147)$ and $49.9 \%(n=28)$ assigned male at birth. A Chi square test confirmed that this difference regarding assigned sex between the binary and non-binary groups was not significant $\left.\left(x^{2}=157\right)=0.43, p=0.51\right)$.

In the binary category, the most common gender identifier was 'Transgender' $(\mathrm{n}=208$, $62.8 \%)$, followed by trans $(n=67,20.2 \%)$, Trans* $27(n=27,8.1 \%)$ and transsexual $(n=16$, $4.8 \%$ ). In the non-binary group, there was a far greater spread of gender identity description. See Table 1.

\section{Insert Table 1 around here}

\section{Differences in anxiety, depression, and self-esteem scale between the binary and non- binary group}

Mann-Whitney $U$ tests were used to compare the differences in scores on the three scales between groups. The analyses indicated that the non-binary group presented with significantly higher levels of anxiety $(U=7072, z=-3.02, p=0.002)$, depression $(U=6731, z=-$ 3.20, $p=0.001)$ and poorer self-esteem $(U=6797, z=-2.94, p=0.003)$ than the binary group. See table 2.

Insert table 2 around here

\section{Differences in levels of social support between the binary and non-binary group}

No significant differences were found between the two groups in the global support score ( $U=7992, z=-1.55, p=0.12$ ), or for any of the subscales; significant others ( $U=9093, z=-.43$, $p=0.68)$, family $(U=8662, z=-.81, p=0.41)$, and friends $(\mathrm{U}=7927, \mathrm{z}=-1.83, \mathrm{p}=.06)$. See table 2 .

Levels of non-suicidal self-injury in the binary and non-binary group. Frequency data showed that, as a percentage, both groups had more people that reported engaging in self- 
injury (non-binary group $=61.4 \%[\mathrm{n}=35]$, binary group $=58.3 \%[\mathrm{n}=193]$ ) compared to not (non-binary group $38.6 \%,[n=22]$, binary group $=41.7 \%,[n=138]$ ). A chi-square test revealed that there were no significant differences in the likelihood of having engaged in selfinjury between the two groups, with alpha level of significance being $p=<0.05\left(\mathrm{x}^{2}(1,388)=\right.$ $.19, p=0.66)$.

\section{$\underline{\text { Discussion }}$}

This study aimed to expand previous research by exploring differences between binary and non-binary identifying young people attending a transgender health service. Until recently, anyone experiencing feelings of gender incongruence was subject to reverse binary gender assumptions (i. e., if one does not identify as male, then they must identify as female, and vice versa). However, the increasing visibility in society of non-binary transgender individuals suggests that this is very frequently not the case (Richards et al., 2016, Clark et al, 2018). Rather, to assume that non-binary and binary transgender individuals experience similar levels of discrimination, are subject to the same kinds of minority stress (Meyer, 2003), and that they therefore experience similar outcomes in terms of support and mental health and wellbeing is fundamentally flawed.

The results of this study with young people suggest that those transgender youth who do not identify with the gender binary suffer from greater anxiety and depression and lower selfesteem than those who identify with it. Furthermore, while there was no difference in the likelihood of engaging in non-suicidal self-injury behaviours between the binary and nonbinary groups, a greater percentage of participants in each group had done so, compared to those who had not. These differences could not easily be attributed to lack of social support, which often predicts mental health symptomology (Davey et al., 2015) as there was no differences between the groups in either overall support, or support from family, friends, or significant others. The finding regarding non-suicidal self-injury behaviour were high, and even higher than previously reported in a similar population (Arcelus et al., 2016). The relatively young age of the sample group may have been an added risk factor for non-suicidal self-injury and could have contributed to the elevated levels of symptomology. 
Interestingly, the distribution of the sex assigned at birth within the group of non-binary transgender individuals differs from non-clinical survey studies with transgender adults. They revealed a ratio of around 2/3 non-binary transgender individuals who were female assigned at birth $(\mathrm{FAAB})$ in relation to $1 / 3$ non-binary transgender individuals who were male assigned at birth (MAAB).

There are several possible explanations for why the non-binary group may be reporting poorer mental health, despite comparable levels of support. Firstly, society in general holds a very deeply rooted view of gender as a binary entity. While there is still a sense of negativity towards those who identify as a gender other than the one assigned at birth, with high levels of discrimination, there is at least a comprehension of the binary to which they subscribe. Non-binary gender, on the other hand, is somewhat less comprehensible to the general public. It is also, at this time, still very much invisible in the media ${ }^{1}$, advertising, films and literature. As a result, those identifying as non-binary are likely to anticipate greater difficulties in navigating their lives within a world where societal understanding and visible role models are lacking. This may contribute to the increased levels of anxiety, depression and low selfesteem that is reported. Relatedly, the potentially more confusing nature of identifying as non-binary at a young age, rather than binary transgender, may contribute to a complex set of issues that include this symptomatology.

Secondly, the emphasis on the binary in everyday society leads to several complex issues for those not identifying as male or female, which may be less simple to resolve than for binaryidentifying transgender people. For example, gendered toilets are an area where the nonbinary person is forced to make a decision from two choices, neither of which may feel right for them. Thus any or either choice could provoke anxiety. Indeed, recent research has found that transgender individuals face many barriers to participating in day to day activities such as sports, due to issues around changing rooms and gendered classes (Jones et al., 2017; Hargie, Mitchell, Somerville, 2017) and non-binary transgender individuals may feel even more excluded from such activities and therefore fail to reap the physical and mental benefits of being physically active. Furthermore, while many people can get accustomed to changing their use of pronouns when a binary identifying person starts to transition, this is not as easy for non-binary individuals and the English and other languages (e. g., German) have little options for non-gendered pronouns. As a result, non-binary transgender teens and adults may

\footnotetext{
${ }^{1}$ Exception: Taylor Mason, a character played by Asia Kate Dillon in Billions, an American television drama series premiered January 2016. Dillon's role on Billions is considered the first non-binary transgender main character on North American television.
} 
be mispronouned and addressed inappropriately more often because of this and being addressed by incorrect pronouns has been found to lead to increased feelings of distress (Eyssel et al., 2017; Guss et al., 2017). Due to lack of understanding, a non-binary transgender individuals may well also meet some resistance when asking friends and family to respect their gender when choosing their language. The Minority Stress Model would suggest that as a minority group, non-binary identifying individuals are at risk from various mental and physical health issues, and the results of this study certainly support this model (Hoy $\square$ Ellis \& Fredriksen $\square$ Goldsen., 2017).

It could also be hypothesised that the explanation for higher levels of mental health problems may be attributed to intrinsic rather than external factors. It could be hypothesised that having a gender identity that it is not easily identifiable can be distressful and confusing for any young person and it may lead to feelings of loneliness and isolation. However, a search for gender identity may be part of wider personality difficulties known to be linked to mental health difficulties.

Finally, although this study did not explore the role of cross-sex hormone use and the data was controlled for this, transgender individuals who start taking hormones have been shown to experience increased mental wellbeing (Gomez-Gil et al., 2012) and individuals who attend for a first assessment having already started to self-mediate have been found to have better mental health than those who have not (Bouman et al., 2017). Thus, future studies may wish to explore the mental health of binary and non-binary transgender youth who have and have not had any medical interventions to confirm this possibility. As the effects and use of hormones has not yet been fully explored among non-binary individuals, the benefits experienced by binary transgender individuals may not be experienced by non-binary transgender individuals and therefore mental wellbeing is likely to remain poorer.

This study found no significances between the two groups when it came to social support. As the minority stress model suggests, minority groups as a whole suffer from lower social support. As both groups are in the minority, it seems as though they both face equal social pressures and lack of support. There was also littler difference found with regards to NSSI levels. Previous studies have suggested that the sex assigned at birth could be a factor in NSSI rates, with female assigned at birth persons showing higher levels of NSSI (Rimes et al, 2017). The results here may suggest that future studies could examine the data not just in 
A limitation to the study is that patients may well take on a more performative gender role in order to access treatment. The effect could be that a small number of non-binary identifying individuals may have reported their gender as binary in nature for the purposes of gaining access to treatments, but actually identify as a non-binary gender otherwise. A strength of this study is that it is one of the first to explore the health of non-binary transgender individuals who have been referred to a gender identity service. Therefore, it is comparing individuals, both binary and non-binary, who have reached a stage where they have sought intervention of some kind. This increases the homogeneity of the sample, compared to a more random sample, collected online for example, that may include individuals at very different stages in their lives. However, a potential limitation is that this study fails to capture the health of those individuals who have not yet been referred or who perhaps never will, for various reasons. Therefore, this study is only generalizable to those who wish the input of transgender health services and may present with higher levels of gender dysphoria than those not involved in services (for a critical discussion see (Eyssel, Koehler, Dekker \& Sehner,Nieder, 2017). The clear issue with a clinical sample is that by their nature, they have already felt the need to seek treatment and therefore must have a higher level of dissatisfaction or mental health symptomology of a non-treatment seeing sample. It must also be noted that a small number of participants may possibly have felt pressure to identify with the binary in order to access treatment and so other non-binary identifying participants could have self-reported as identifying with the binary. The number of non-binary participants (which have included 7 people questioning their gender) is still small and future studies may benefit from involving larger number of participants. There is also a comparatively different sample size between the binary and non-binary groups which must be acknowledged as a possible limitation. Notwithstanding, this study provides clear evidence that non-binary transgender youth suffer from greater mental wellbeing problems than binary transgender youth. As this study is mainly descriptive and based on the non-binary population attending transgender health services, future studies could explore mental health problems using a qualitative methodology in order to understand better factors influencing the development of mental health difficulties in this population. 


\section{Conclusion}

To date, research, especially based on clinical samples, has failed to represent the non-binary population. This study has shown that non-binary and binary transgender individuals are not a heterogeneous group, that non-binary youth have a different distribution in term of sex assigned at birth compared with non-binary adults and suffer from poorer mental health than their binary identifying counterparts. Future research needs to seek to understand why nonbinary youth are more greatly affected and use this to inform the development of interventions that can help educate the general population and support gender questioning individuals. 
Budge, S. L., Adelson, J. L., \& Howard, K. A. (2013). Anxiety and depression in transgender individuals: the roles of transition status, loss, social support, and coping. Journal of consulting and clinical psychology, 81(3), 545.

Cohen, J. (1988). Statistical power analysis for the behavioral sciences . Hilsdale. NJ: Lawrence Earlbaum Associates, 2.

Clara, I. P., Cox, B. J., Enns, M. W., Murray, L. T., \& Torgrudc, L. J. (2003). Confirmatory factor analysis of the multidimensional scale of perceived social support in clinically distressed and student samples. Journal of personality assessment, 81(3), 265-270.

Clark, B. A., Veale, J. F., Townsend, M., Frohard-Dourlent, H., \& Saewyc, E. (2018). Non-binary youth: Access to gender-affirming primary health care. International Journal of Transgenderism, 1-12. 
Collin, L., Reisner, S. L., Tangpricha, V., \& Goodman, M. (2016). Prevalence of transgender depends on the "case" definition: a systematic review. The journal of sexual medicine, 13(4), 613-626.

Cecil, H., Stanley, M.A., Carrion, P.G., \& Swann, A. (1995). Psychometric properties of the MSPSS and NOS in psychiatric outpatients. Journal of Clinical Psychology, 51, 593-602.

Claes, L., Bouman, W.P., Witcomb, G.L., Thurston, M., Fernandez-Aranda, F, \& Arcelus, J. (2015). Non-suicidal self-injury in trans people: associations with psychological symptoms, victimization, interpersonal functioning, and perceived social support. Journal of Sexual Medicine, 12(1), 168-179.

Claes, L., \& Vandereycken, W. (2007). The Self-Injury Questionnaire-Treatment Related (SIQ-TR): Construction, reliability, and validity in a sample of female eating disorder patients. In P.M. Goldfarb (Ed.), Psychological Tests and Testing Research Trends (pp. 111-139). New York: Nova Science Publishers.

Dahlem, N.W., Zimet, G.D., \& Walker, R.R. (1991). The Multidimensional Scale of Perceived Social Support: a confirmation study. Journal of Clinical Psychology, 47, 756-761.

Dargie, E., Blair, K. L., Pukall, C. F., \& Coyle, S. M. (2014). Somewhere under the rainbow: Exploring the identities and experiences of trans persons. The Canadian Journal of Human Sexuality, 23(2), 60-74.

Davey, A., Arcelus, J., Meyer, C., \& Bouma, W.P. (2016). Self-injury among trans individuals and matched controls: prevalence and associated factors. Health and Social Care Community, 24(4), 485-494.

Eyssel, J., Koehler, A., Dekker, A., Sehner, S., \& Nieder, T. O. (2017). Needs and concerns of transgender individuals regarding interdisciplinary transgender healthcare: A non-clinical online survey. PloS one, 12(8), e 0183014. 
Gómez-Gil, E., Zubiaurre-Elorza, L., Esteva, I., Guillamon, A., Godás, T., Almaraz, M. C., ... \& Salamero, M. (2012).). Hormone-treated transsexuals report less social distress, anxiety and depression. Psychoneuroendocrinology, 37(5), 662-670.

Gross, M. (2016). Transitions to new concepts of gender. R141-R143.

Guss, C. E., Woolverton, A., Borus, J., Reisner, S. L., Austin, S. B., \& Katz-Wise, S. L. (2017). “Just Step Up:” A Qualitative Study of Transgender Adolescents' Experiences in Primary Care. Journal of Adolescent Health, 60(2), S28-S29.

Hargie, O. D., Mitchell, D. H., \& Somerville, I. J. (2017). 'People have a knack of making you feel excluded if they catch on to your difference': Transgender experiences of exclusion in sport. International Review for the Sociology of Sport, 52(2), 223-239.

Hage, J. J. and R. B. Karim (2000). "Ought GIDNOS get nought? Treatment options for nontranssexual gender dysphoria." Plastic and Reconstructive Surgery 105(3): 1222-1227.

Heylens, G., Elaut, E., Kreukels, B. P., Paap, M. C., Cerwenka, S., Richter-Appelt, H., ... \& De Cuypere, G. (2014). Psychiatric characteristics in transsexual individuals: multicentre study in four European countries. The British Journal of Psychiatry, 204(2), 151-156.

Hoy $\square$ Ellis, C. P., \& Fredriksen $\square$ Goldsen, K. I. (2017). Depression among transgender older adults: General and minority stress. American journal of community psychology, 59(3-4), 295-305.

Jones, B. A., Arcelus, J., Bouman, W. P., \& Haycraft, E. (2017). Sport and transgender people: a systematic review of the literature relating to sport participation and competitive sport policies. Sports Medicine, 47(4), 701-716.

Kessler, R. C., Berglund, P., Demler, O., Jin, R., \& Walters, E. E. (2005). Lifetime prevalence and age of-onset distributions of DSM-IV disorders in the national comorbidity survey replication. Archives of General Psychiatry, 62, 593-602.

Laye-Gindhu, A., \& Schonert-Reichl, K. (2005). Nonsuicidal self-harm among community adolescents: Understanding the "Whats" and "Whys" of self-harm. Journal of Youth and Adolescence, 34(5), 447-457. 
Marshall, E., Claes, L., Bouman, W. P., Witcomb, G. L., \& Arcelus, J. (2016). Non-suicidal selfinjury and suicidality in trans people: a systematic review of the literature. International Review of Psychiatry, 28(1), 58-69.

Meier, S. C., Pardo, S. T., Labuski, C., \& Babcock, J. (2013). Measures of clinical health among female-to-male transgender persons as a function of sexual orientation. Archives of Sexual Behavior, 42(3), 463-474.

METRO Youth Chances (2014). Survey of 16-25 year olds: First Reference Report. London: METRO.

Meyer, I.H. (2003). Prejudice, social stress, and mental health in lesbian, gay and bisexual populations: Conceptual issues and research evidence. Psychological Bulletin, 129; 674-697.

Millet, N., Longworth, J., \& Arcelus, J. (2017). Prevalence of anxiety symptoms and disorders in the transgender population: A systematic review of the literature. International Journal of Transgenderism, 18(1), 27-38.

Nieder, T. O., \& Richter-Appelt, H. (2011). Tertium non datur-either/or reactions to transsexualism amongst health care professionals: the situation past and present, and its relevance to the future. Psychology \& Sexuality, 2(3), 224-243.

Nuttbrock, L., Rosenblum, A., \& Blumenstein, R. (2002). Transgender identity affirmation and mental health. International Journal of Transgenderism, 6(4), 97-103.

Office for National Statistics, Measuring National Well-being: Domains and Measures (2017), London, Office for National Statistics

Rimes, K. A., Goodship, N., Ussher, G., Baker, D., \& West, E. (2017). Non-binary and binary transgender youth: comparison of mental health, self-harm, suicidality, substance use and victimization experiences. International Journal of Transgenderism, 1-11.

Richards, C. (2017). In Bouman, W.P. \& Arcelus, J. (Eds.). The Transgender Handbook: A Guide for Transgender People, Their Families, and Professionals. New York, NY: Nova Publishers, 2017.

Richards, C., Bouman, W. P., Seal, L., Barker, M. J., Nieder, T. O., \& T’Sjoen, G. (2016). Nonbinary or genderqueer genders. International Review of Psychiatry, 28(1), 95-102. 
Richards, C., Bouman, W. P., \& Barker, M. J. (Eds.). (2017). Genderqueer and non-binary genders. Springer.

Robins, R. W., Hendin, H. M., \& Trzesniewski, K. H. (2001). Measuring global self-esteem: Construct validation of a single-item measure and the Rosenberg Self-Esteem Scale. Personality and social psychology bulletin, 27(2), 151-161.

Rosenberg, M. (1965). Rosenberg self-esteem scale (RSE). Acceptance and commitment therapy. Measures package, 61, 52.

Rotondi, N. K., Bauer, G. R., Scanlon, K., Kaay, M., Travers, R., \& Travers, A. (2013). Nonprescribed hormone use and self-performed surgeries:"do-it-yourself" transitions in transgender communities in Ontario, Canada. American Journal of Public Health, 103(10), 1830-1836.

Skrapec, C.,\& MacKenzie, K.R. (1981). Psychological self-perception in male transsexuals, homosexuals, and heterosexuals. Archives of Sexual Behavior, 10(4), 357-70.

Södersten, M., Nygren, U., Hertegård, S., \& Dhejne, C. (2015). Interdisciplinary program in Sweden related to transgender voice. SIG 3 Perspectives on Voice and Voice Disorders, 25(2), 87-97.

Stocks, T. (2015). To what extent have the rights of transgender people been underrealized in comparison to the rights of lesbian, gay, bisexual, and queer/questioning people in the United Kingdom? International Journal of Transgenderism, 16(1), 1-35.

Vocks, S., Stahn, C., Loenser, K., \& Legenbauer, T. (2009). Eating and body image disturbances in male-to-female and female-to-male transsexuals. Archives of Sexual Behavior, 38(3), 364377.

Wiseman, M., \& Davidson, S. (2012). Problems with binary gender discourse: Using context to promote flexibility and connection in gender identity. Clinical Child Psychology and Psychiatry, 17(4), 528-537.

Witcomb, G. L., Bouman, W. P., Claes, L., Brewin, N., Crawford, J. R., \& Arcelus, J. (2018). Levels of depression in transgender people and its predictors: Results of a large matched control 
study with transgender people accessing clinical services. Journal of Affective Disorders. https://doi.org/10.1016/j.jad.2018.02.051

Worthen, M. G. (2016). Hetero-cis-normativity and the gendering of transphobia. International Journal of Transgenderism, 17(1), 31-57.

Zigmond, A. S., \& Snaith, R. P. (1983). The hospital anxiety and depression scale. Acta Psychiatrica Scandinavica, 67(6), 361-370.

Zimet, G. D., Dahlem, N. W., Zimet, S. G., \& Farley, G. K. (1988). The multidimensional scale of perceived social support. Journal of Personality Assessment, 52, 30-41.

Zucker, K. J. (2017). Epidemiology of gender dysphoria and transgender identity. Sexual health. 
Table 1. Gender identity of non-binary participants $(\mathbf{n}=\mathbf{5 7})$

\begin{tabular}{|l|l|}
\hline & Total and percentage \\
\hline Non Binary & $42(73.6 \%)$ \\
\hline Neutral & $6(10.5 \%)$ \\
\hline Agender & $3(5.2 \%)$ \\
\hline Bigender & $3(5.2 \%)$ \\
\hline Gender fluid & $3(5.2 \%)$ \\
\hline & \\
\hline
\end{tabular}


Table 2. Total mean scores and standard deviations (SD) for the HADS- Anxiety, HADS- Depression, Rosenberg Self-Esteem Scale, and the Multidimensional Scale of Perceived Social Support for both binary and non-binary groups .

\begin{tabular}{|l|c|c|}
\hline & $\begin{array}{c}\text { Binary } \\
(\mathrm{n}=331)\end{array}$ & $\begin{array}{c}\text { Non-binary } \\
(\mathrm{n}=57)\end{array}$ \\
\hline Anxiety & $9.71(4.47)$ & $9.00(4.00)^{*}$ \\
\hline Depression & $7.06(3.73)$ & $11.02(6.01)^{*}$ \\
\hline Self-esteem & $13.5(0.32)$ & $21.25(6.17)$ \\
\hline $\begin{array}{l}\text { Support from significant } \\
\text { others }\end{array}$ & $21.47(6.38)$ & $17.88(6.63)$ \\
\hline Support from Family & $18.54(6.38)$ & $19.14(6.43)$ \\
\hline Support from Friends & $20.69(6.07)$ & $58.26(13.45)$ \\
\hline Overall support & $60.80(13.38)$ & \\
\hline$p<0.05$ & & \\
\hline
\end{tabular}

